\title{
A portable micro-based system for the study of associated movements
}

\author{
ROBIN MORRIS, RICHARD HELMER, and HASKEL HOINE \\ Trinity University, San Antonio, Texas 78284
}

\begin{abstract}
This paper describes a microcomputer-based system for the on-line study of associated movements. The system is portable, provides hard copy, includes a backup system, is relatively inexpensive, can be used with simple or choice reaction time paradigms, and is simple to operate.
\end{abstract}

An associated movement is an involuntary movement of the contralateral extremity when its opposite member is performing a voluntary act. The measurement of associated movements is incorporated into many neurological evaluations of children. The presence of these movements has been observed in healthy young children, as well as in older children who have neurological problems.

Differences in technique, combined with a high degree of subjectivity, have yielded a good deal of imprecision to the results of previous research. Stern, Gold, Hoine, and Barocas (1976) attempted to develop a more precise and objective technique for the assessment of associated movements. This paper describes a portable, microcomputer-based system for the on-line study of associated movements, based on procedures described by Hoine (1976) and Stern (Note 1).

The basic paradigm involves a series of reaction time tasks, with increasing levels of complexity: bilateral simple reaction time, unilateral simple reaction time, choice reaction time, and incompatible (reverse) choice reaction time. While the child is performing the appropriate response to stimuli with one hand, the movements in both hands are being monitored and recorded. The relationship of the voluntary movements required by the task and any involuntary movements occurring in the other hand during that time are the variables of interest.

An example of a simple reaction time trial involving the left hand will help describe the system's function. The child is asked to place the left and right index fingers on the left and right "set" sensors (Figure 1). When the left LED goes on, the child is to move the left index finger to the left "goal" sensor. When the LED goes off, the child is to return the finger back to the left "set" sensor. After this return, an interval occurs in

The authors wish to thank Brian Sanders and John Howland of the Computer Science Department for their invaluable assistance. We also wish to acknowledge Nancy Sherman for building the stimulus-response panel and Douglas Eddy for his review of this project and this report. Requests for reprints should be sent to Haskel Hoine, Department of Psychology, Trinity University, 715 Stadium Drive, San Antonio, Texas 78284. which movements are continually monitored. During the entire trial, any movements made by the right index finger are recorded, as well as movements made by the left index finger.

The purpose of the system is to present stimuli, time stimuli and intertrial intervals, monitor movement in either index finger, record the type and time of a movement, and print out a session's results. Additionally, two important requirements of the research are met by the system: It is portable and includes a backup capability for recording data if the microcomputer should fail.

The portability feature allows for easy transportation to provide on-line capability in schools, hospitals, and other child-oriented programs. Each component of the system is easily handled by one person and fits into a small physical area. This feature helps widen subject population availability.

The backup feature allows for continuous data collection when the microcomputer fails. This feature of the system is important because acquiring subjects from a child population is becoming increasingly difficult. Losing data or being unable to test available subjects due to equipment failure is a problem all researchers would like to avoid. All stimulus presentation and response recording can be done without the microprocessor. Analog codes for stimuli and all responses are fed into an FM tape recorder. The session's tapes can later be analyzed on the micro, or any other appropriately programmed computer. In this way, data gathering should rarely be interrupted. Of course, on-line analysis is much preferred because off-line analysis of the analog data requires at least twice as much total time per subject.

\section{HARDWARE}

The system is built around a Digital Group Z-80 microcomputer (Figure 1). The micro is equipped with 24K RAM, four 8-bit parallel I/O ports, and a $1,000-\mathrm{Hz}$ external clock circuit. The micro is connected to a Hitachi portable television (P-05) for video display, an ASCII keyboard, a Southwest Technical Products 


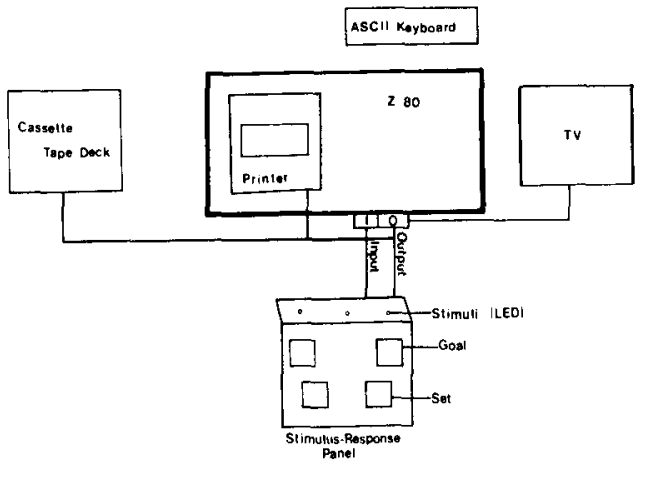

Figure 1. Associated movement system.

Corporation (SWTPC) 40-character line printer (PR-40), an A. R. Vetter four-channel analog cassette tape deck (C-4), and a stimulus presentation and response panel built in-house.

The stimulus-response panel includes the necessary components for the control of stimuli via an output port. It also provides the appropriate TTL levels to the Z-80's input port for the monitoring of responses during the experimental session. The panel is designed to output analog stimulus and response codes to the cassette recorder as part of the backup system. A stimulus code is placed on one channel, while left and right movements are coded on two other channels. Finally, the panel provides the controls for manual stimulus presentation if required.

The cassette deck has multiple purposes. Initially, the microprocessor's operating system and software are loaded using it. During the experimental session, the deck provides the system backup as previously noted. After the session is over, it is used for the storage of digitized data.

The total system cost, including the backup components, is under $\$ 3,000$. The cost of the cassette tape deck accounted for a little less than half the cost of the entire system. The printer (\$250) and television (\$90) were two of the least expensive components. We feel that the small added savings brought about by the purchasing of kits (Z-80 and printer) was not worth the cost in time to build and debug them. We suggest that others buy preassembled and tested components if they lack time, experience, or the technical skills required.

\section{SOFTWARE}

The total system was written in Digital Group's Maxi BASIC and assembler. The program is simple and is easily used by minimally trained individuals. The software can be broken down into four components: the main core program, the data analysis subroutine, a timing routine, and a printer routine.

The printer and timing routines were both written in assembler and added to the Z-80 operating system provided by Digital Group. The printing routine uses one
1/O port for sending characters to the printer's 40 character buffer. The SWTPC printer prints a line when its buffer is filled or upon receiving a carriage-return command.

The timing routine uses a feature of Digital Group's BASIC called the CALL function. This function allows the transfer of a decimal number between routines written in BASIC or assembler. The timing routine receives two values passed to it from the core program. The values are loaded into a memory location and decremented by the routine's interrupt handler. Interrupts are produced by the $1,000-\mathrm{Hz}$ clock circuit. One value controls stimulus duration, while the other controls the duration of the entire trial. The values in these locations are returned to the BASIC core program to obtain accurate millisecond latencies.

The main core program and analysis subroutine are both written in BASIC. The main routine reads values from the experimenter initializing the timing and stimulus parameters. Upon trial initiation, it turns the stimulus on and continually monitors the input port connected to the four response sensors (each represented as a bit). When any changes occur, the program records the stimulus condition, the new state, and the time of the movement. Digital Group's BASIC provides easy input and output functions for these purposes. The statement OUT 2, X turns on a bit(s) on Port 2, which illuminates an $\operatorname{LED}(\mathrm{s})$. The variable $X$ is the number corresponding to the needed bit(s) in an 8-bit word. A similar procedure is used to poll an input port. The statement $Y=\operatorname{INP}(3)$ assigns the current value of the 8-bit word connected with Input Port 3 to the variable Y. In our system, when a finger contacts a sensor, it registers on a specific bit as "on" (1). When there is no contact, as when a finger lifts off a sensor, the appropriate bit reads "off" $(0)$. In this way, the system can monitor finger contact with a particular sensor(s).

At the end of a trial series, all movements for each trial are examined by the analysis subroutine. This subroutine provides forward and return reaction and movement times. It also analyzes error movements (responses with wrong finger, no response, etc.), reports the number and duration of associated movements, and reports the occurrence of extraneous movements (unrelated to the stimulus) in either hand. Finally, it performs the appropriate data averaging across a series of trials for each subject.

\section{SUMMARY}

A system was described that provides for an objective measure of associated movements for clinical research purposes. It is portable, provides hard copy, includes a backup system, is relatively inexpensive as on-line systems go, can be used for simple- and multiple-choice reaction time paradigms with little modification, has excellent BASIC function for $\mathrm{I} / \mathrm{O}$ and interaction with 
assembler routines, and is simple enough to operate with little training.

\section{REFERENCE NOTE}

1. Stern, J. A. Response inhibition: Its role in child development and aging. Paper presented at the Missouri Psychological Association, November 1976.

\section{REFERENCES}

HoINE, H. Motor overflow and the speed of reaction in children. Unpublished doctoral dissertation, Washington University at St. Louis, December 1976.

Stern, J. A., Gold, S., Hoine, H., \& Barocas, V. S. Toward a more refined analysis of the "overflow" or "associated movement" phenomenon. In D. V. S. Sankar (Ed.), Mental health in children (Vol. 2). New York: PJD Publications (whole monograph), 1976. 